\title{
EXPERIMENTAL RESULTS ON SINGLE FLUX QUANTUM LOGIC
}

\author{
S. P. Benz, C. J. Burroughs, and C. A. Hamilton \\ National Institute of Standards and Technology \\ Division 814.03, 325 Broadway, Boulder, CO 80303
}

Abstract--We have optimized the design and calculated the margins for a number of single flux quantum (SFQ) logic elements including AND, OR, XOR, Splitter, DC-to-SFQ converter, and SFQ-to-DC converter. These are the fundamental building blocks necessary to construct more complex logic functions such as the half adder, and full adder. Experimental tests of the primary gates, the AND, OR, XOR, and splitter, were made by imbedding each test gate between DC-to-SFQ converters at the inputs and SFQ-to-DC converters at the outputs. Automated testing of each circuit was used to determine functionality, optimum bias levels, and margins. The experimental bias current margins for each gate are consistent with the simulations. This is the first experimental functional confirmation of these SFQ logic gates.

\section{INTRODUCTION}

The rapid single flux quantum (RSFQ) logic family was proposed by Likharev, Mukhanov, and Semenov [1-3] as a low-power, high-speed, Josephson-junction-based alternative to semiconductor logic. Since RSFQ logic operates with dc power and is self resetting, it overcomes the main performance difficulties of Josephson voltage-state logic: crosstalk and slow resetting speed. It also has the potential for a higher operating temperature than voltage-state logic through the use of nonhysteretic high- $\mathrm{T}_{\mathrm{c}}$ junctions. Hamilton and Gilbert [4] optimized the parameters for the AND, OR, XOR, and splitter elements of the RSFQ logic family. By maximizing the critical margin, which is defined as the smallest margin, they found the critical margin for each circuit to be greater than about $30 \%$ (see Table 1 ).

In this paper we present the first experimental verification of these RSFQ logic gates. We describe the design and optimization of the input and output circuits, the circuit layout and low- $\mathrm{T}_{\mathrm{c}}$ fabrication process, and the experimental tests. Comments will be made throughout the paper on the implications and prospects of using these primary circuit elements for constructing more complex functions such as the half adder and full adder.

\section{TEST CIRCUIT DESIGN AND OPTIMIZATION}

The inputs and outputs of RSFQ circuits are single flux quantum (SFQ) pulses with a typical amplitude of $1 \mathrm{mV}$ and a duration of 2 ps. Since these pulses can neither be generated nor directly detected with room temperature electronics, it is necessary to imbed the RSFQ circuit elements between $\mathrm{DC} / \mathrm{SFQ}$ and SFQ/DC converters [5]. The input/output (I/O) circuits used in these experiments are shown in Fig. 1. In the DC/SFQ circuit I2 biases junction J2 below its critical

Manuscript received August 24, 1992.

U.S. Government work not protected by U.S. copyright. current. Application of the input current $\mathrm{I}_{\text {in }}$ causes an SFQ transition ( $2 \pi$ phase advance) in $\mathrm{J} 2$ launching an SFQ pulse into the test circuit and trapping a circulating current in loop L1-J1-J2. The magnetically coupled, unshunted read SQUID J3-L2-J4 detects the circulating current in L1 confirming correct operation. Reversing $I_{\text {in }}$ causes an SFQ transition in $\mathrm{J} 1$ resetting the circuit to its initial state.

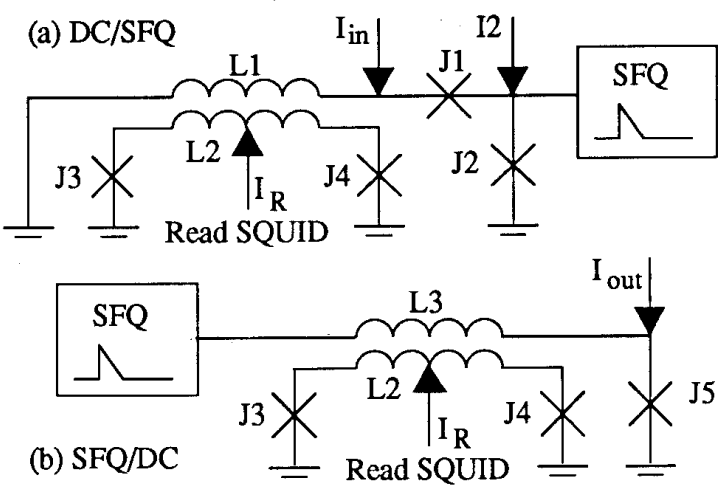

Fig. 1 (a) DC/SFQ converter input circuit. (b) SFQ/DC converter output circuit. The standard experimental parameters were $\mathrm{I}_{\mathrm{C}} 1=177 \mu \mathrm{A}, \mathrm{I}_{\mathrm{C}} 2=126 \mu \mathrm{A}, \mathrm{L} 1=$ $\mathrm{L} 3=17.8 \mathrm{pH}, \mathrm{I}_{\mathrm{in}}= \pm 165 \mu \mathrm{A}, \mathrm{I} 2=115 \mu \mathrm{A}, \mathrm{I}_{\mathrm{C}} 5=$ $133 \mu \mathrm{A}, \mathrm{I}_{\text {out }}=120 \mu \mathrm{A}, \mathrm{I}_{\mathrm{C}} 3=\mathrm{I}_{\mathrm{C}} 4=75 \mu \mathrm{A}, \mathrm{L} 2=$ $13.8 \mathrm{pH}$. The mutual inductance between $\mathrm{L} 1$ and $\mathrm{L} 2$ was $8.9 \mathrm{pH}$, and $\beta_{\mathrm{c}}=1$ and $\mathrm{C} / \mathrm{I}_{\mathrm{c}}=3.7 \mathrm{pF} / \mathrm{mA}$ for all shunted junctions.

In the SFQ/DC converter an SFQ pulse at the input traps a circulating current in the loop L3-J5 and the junction in the preceding stage (not shown). The circulating current in L3 is detected by the read SQUID. Application of $\mathrm{I}_{\text {out }}$ causes an SFQ transition in J5 resetting the circuit. We chose L1 = L3 so identical read SQUIDs could be used in the input and output circuits. The operation of the primary RSFQ gates has been described previously [1-3].

To ensure successful operation, the input and output parameters were optimized specifically for each RSFQ gate ${ }^{1}$ using the same method described in [4]. First, the RSFQ gate parameters were fixed at the optimized values found by Hamilton and Gilbert [4]. Margins were calculated for each parameter of the entire test circuit (the primary gate including the input and output circuits) and the critical margin determined. Then each $\mathrm{I} / \mathrm{O}$ parameter was adjusted one by one in whichever direction increased the critical margin, while still

${ }^{1}$ An additional junction-inductor stage $(100 \mu \mathrm{A}, 9.7 \mathrm{pH}, 108 \mu \mathrm{A}$ bias) was added to the AND gate A and B inputs (see Ref. [4]) to separate the stored flux quanta in the input stage (L1-J1-J2) from that in the AND gate (J1-L1-J5-J7) prior to the clock pulse. 
keeping the primary gate parameters fixed. The resulting critical margin for each test circuit with optimized I/O parameters is shown in Table 1. In all cases, the critical margin parameter was $\mathrm{I}_{\text {in }}$ of the DC/SFQ converter. Note that the critical margins are reduced, except for the AND gate ${ }^{2}$ from those of the primary gates as a result of loading from the $\mathrm{I} / \mathrm{O}$ circuits.

Table 1. Simulated RSFQ circuit Critical Margins

\begin{tabular}{|c||c|c|c|}
\hline $\begin{array}{c}\text { RSFQ } \\
\text { Gate }\end{array}$ & $\begin{array}{c}\text { Primary } \\
\text { Gate [4] }\end{array}$ & $\begin{array}{c}\text { Optimized I/O } \\
\text { Test Circuit }\end{array}$ & $\begin{array}{c}\text { Standard I/O } \\
\text { Test Circuit }\end{array}$ \\
\hline Splitter & $47 \%$ & $46 \%$ & $32 \%$ \\
\hline AND & $32 \%$ & $40 \% 2$ & $30 \%$ \\
\hline OR & $35 \%$ & $29 \%$ & $25 \%$ \\
\hline XOR & $30 \%$ & $21 \%$ & $16 \%$ \\
\hline
\end{tabular}

In keeping with our goal of using standardized SFQ elements, we next determined the margins of the gates for a standard set of $\mathrm{I} / \mathrm{O}$ parameters to be used in the experiments. The standard I/O parameters are given in the caption of Fig. 1. The use of standard $\mathrm{H} O \mathrm{O}$ parameters for the test circuits reduces the critical margins even further.

\section{LAYOUT AND FABRICATION}

The layout for the primary RSFQ gates with their I/O circuits is relatively straightforward because only a single clock pulse is required and the connections between junctions are short and can easily be made to satisfy the typical $5-8 \mathrm{pH}$ design requirement. The layout of the more complex half and full adder circuits is substantially more difficult for two reasons: (1) Some of the interconnection lengths are too long to achieve with a 5-8 $\mathrm{pH}$ inductor, and (2) each gate requires a clock pulse which is precisely timed with respect to the other gates. To satisfy these requirements, Josephson transmission lines were used in several key places to transmit SFQ pulses through lines of greater than $8 \mathrm{pH}$ and to achieve the appropriate clock timing [4].

The timing requirements and extraordinary geometry dependence of RSFQ logic make its layout significantly more difficult than voltage-state logic where elements can be interconnected with superconducting striplines of unspecified length. The requirement for shunted SIS tunnel junctions which demand considerably more chip area than unshunted junctions, also adds geometrical constraints. The development of critically damped, unshunted, high-current density junctions using submicrometer lithography would solve this problem.

Satisfying the geometrical and timing constraints for the half and full adders required an iterative layout process. First the primary gates were designed and laid out independently. Then the AND, the XOR, and three splitters were connected to construct the half adder with appropriate readjustments to make all interconnections fit. Finally, two half adders, three Josephson transmission lines, and two more splitters were connected together and restructured again to complete the full

2The optimized critical margin for the AND gate test circuit increased because of the additional $A$ and $B$ input cells (see Footnote 1). adder. These final versions of the primary gates were used in the test circuits so they would be identical to the internal gates of the full adder test circuit. Unfortunately the constraints required four different versions of the splitter to complete the full adder layout. These test circuit layouts required considerable adjustment of the widths of inductors and the positions of junctions to match both the circuit design and the process design rules. Without any concem for circuit compaction, the final area of the individual test circuits was approximately $310 \mu \mathrm{m}^{2}$, and the full adder test circuit was 1 $\mathrm{mm}^{2}$. Both circuits could probably be reduced to $30 \%$ of this size, with sufficient persistence.

The circuits were laid out on a $1 \times 1 \mathrm{~cm}^{2}$ chip which has 96 contact pads. All test circuits, including those for the half adder and full adder, were on the same chip. A typical test circuit schematic is shown in Fig. 2 for the OR gate. DC power was supplied on a common bus line to all internal primary gate biases and the 12 bias of each input circuit. The bus was designed to operate at $1 \mathrm{mV}$ so that each intemal bias was determined by a resistor to the bus. Process variations common to all resistors can be compensated by changing the bus voltage, but any variation in the resistor ratios has a severe effect on the other circuit margins. The margins that can be determined experimentally are the those of the input and output bias lines and the bus line.

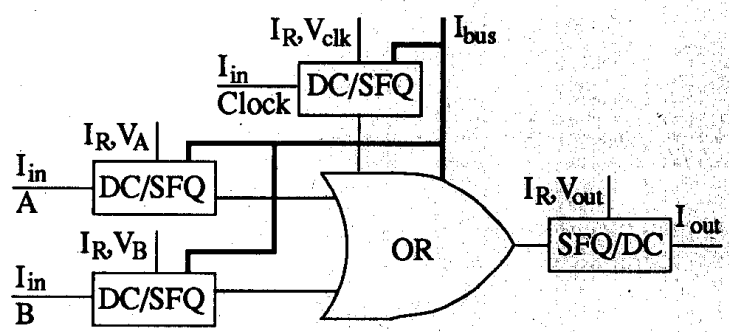

Fig. 2 SFQ OR gate test circuit showing the experimentally measurable biases. The fat line is the common bus to each element.

The circuits were fabricated using an 8-level process: $\mathrm{Nb}$ $\mathrm{Al}_{2} \mathrm{O}_{3}-\mathrm{Nb}$ trilayer junctions with current density $560 \mathrm{~A} / \mathrm{cm}^{2}$ and thicknesses $220 \mathrm{~nm}(\mathrm{BE}), 10 \mathrm{~nm}(\mathrm{Al})$, and $140 \mathrm{~nm}$ (CE); AuIn $_{2}$ resistors with $1 \Omega$ sheet resistance; $300 \mathrm{~nm}$ thick first $\mathrm{SiO}$ insulator (S1); $450 \mathrm{~nm}$ thick $\mathrm{Pb}$-In-Au alloy wiring layer (WR); $600 \mathrm{~nm}$ thick second SiO insulator (S2); and $1 \mu \mathrm{m}$ thick $\mathrm{Pb}$-In-Au alloy ground plane (GP) over the entire circuit.

Table 2. Test structures on the SFQ Logic chip

\begin{tabular}{|lc|}
\hline \multicolumn{1}{|c}{ Circuit } & No. of Junctions \\
\hline Series Array $\left(11.5 \mu \mathrm{m}^{2}\right)$ & 400 \\
Series Array $\left(36 \mu \mathrm{m}^{2}\right)$ & 400 \\
Read SQUID & 2 \\
Inductance SQUID (WR-GP) & 2 \\
Inductance SQUID (BE-GP) & 2 \\
Capacitance $\left(9 \mu \mathrm{m}^{2}\right)$ & 2 \\
Shunted Junctions $(0.5-3$ squares) & 6 \\
3-Resistors $\left(6 \times 6,6 \times 30,50 \times 50 \mu \mathrm{m}^{2}\right)$ & - \\
4-Interlevel Contact Tests & - \\
\hline
\end{tabular}


Thirty different square junctions with areas ranging from 9 $\mu \mathrm{m}^{2}$ to $38 \mu \mathrm{m}^{2}$ were required to implement all of the circuits and other test structures on the chip. The test structures are listed in Table 2. A systematic error in junction critical currents resulting from etchback of the counterelectrodes was found using the 400 -junction series arrays. New masks were made to account for the etchback. Another systematic error which was not accounted for at the original layout, and has yet to be corrected, resulted from contact resistance, typically 0.25 $\Omega$, between the resistors and the $\mathrm{Nb}$ base electrode. This has a considerable effect since some of the resistors (shunts) have design values as small as $0.5 \Omega$.

The on-chip critical current uniformity of the smallest junctions in the SFQ test circuits was found to be better than $1 \%$ at 1 standard deviation as measured from the small junction series array. The inductor and resistor uniformities, again measured from test structures, were both better than $3 \%$ across the $5.1 \mathrm{~cm}$ diameter wafer. The biggest fabrication difficulty encountered was not across-chip uniformity, but simultaneously achieving the desired target values for current density, resistance and inductance. The test structures were essential for improving the process control of these parameters to better than a few percent.

\section{EXPERIMENTAL TEST RESULTS}

The experimental testing of these circuits required a sophisticated system to manipulate the multiple input and output biases and to monitor their flux states. The NIST SFQ Test System was designed specifically for testing SFQ circuits and is described in detail elsewhere [6]. This system enabled the initial bias parameters to be determined interactively and the margin search and optimization to be fully automated.

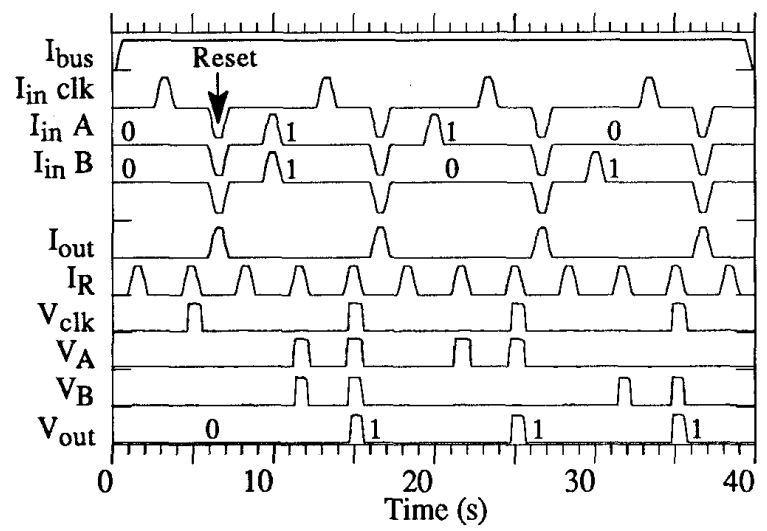

Fig. 3 Experimental automated logic test results for the SFQ OR gate. The top six traces show the bias conditions and the bottom four traces show the read SQUID voltage states. A high voltage when $I_{R}$ is high indicates the presence of a stored SFQ.

The circuits were first made to operate by adjusting the bus, input, and output biases. The input and output biases were found for a given bus bias by determining threshold characteristics for the inputs and outputs using the read SQUIDs and either $I_{\text {in }}$ or $I_{\text {out }}$ as a control line. Offset currents to $I_{\text {in }}$ and $I_{\text {out }}$ were sometimes used to correctly position the input and output biases in a threshold lobe. $I_{R}$ was fixed midway between the SQUID threshold current for a 1 and 0 in the I/O circuit and was typically $100 \mu \mathrm{A}$.

Once these initial operating points and read SQUID thresholds were found, the bias conditions were checked for correct logic operation. The input sequence used to test the four logic conditions, no input, A and B, A only, and finally B only, is plotted in Fig. 3 with the read SQUID voltages and shows the correct logic operation for the experimental $O R$ gate. The currents are all normalized to their bias values, and the voltages are normalized to $2.8 \mathrm{mV}$.

The input, output, and bus current margins were determined and optimized using the same method as the simulations. The margins were searched up to a maximum of $\pm 70 \%$. The automated test circuit usually completed the margin optimization within 24-48 hours. The optimized experimental margins for four test circuits on the same chip are shown in Fig. 4 and the critical margins are listed in Table 3.

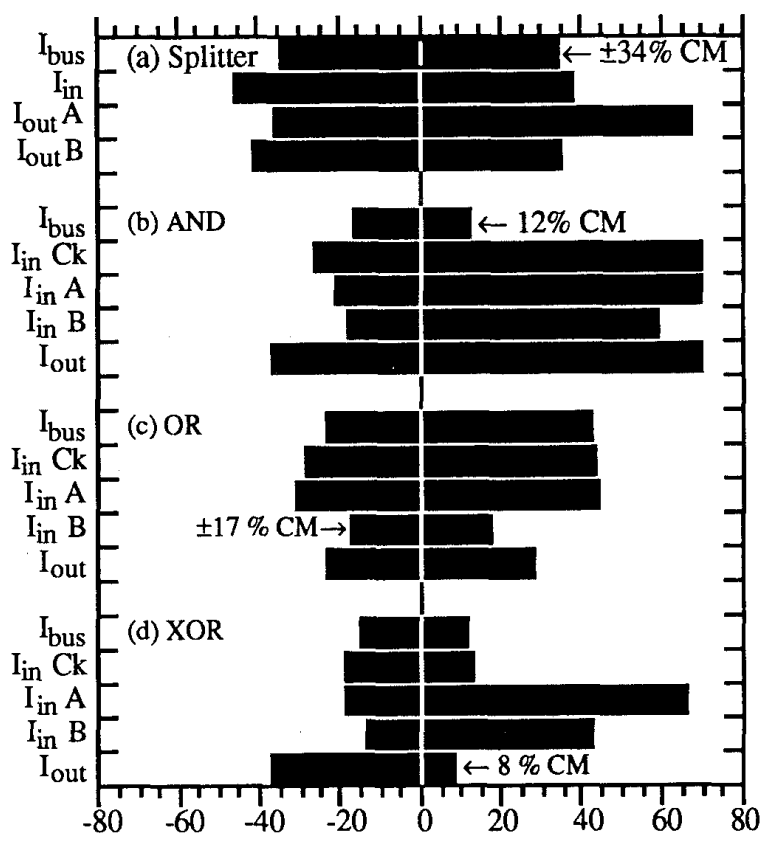

Fig. 4 Experimental RSFQ logic margins: (a) Splitter, (b) AND, (c) OR, and (d) XOR.

The critical margin for the OR test circuit, $I_{\text {in }} B$, was unexpected since it is laid out essentially symmetrically with respect to $\mathrm{A}$ and $\mathrm{B}$. This lower margin for $\mathrm{B}$, which is also observed for the other chips from this wafer, may be due to a mask or exposure defect. The parameter with the smallest total margin was typically the bus bias, and that the gate with the smallest bus line margin is the AND gate. This is 
probably a result of the systematic error in the fabricated resistor ratios due to contact resistance. The total bus line margin seems to scale inversely with the number of biases to the bus. Given this trend it is not surprising that we were unable to make the half and full adder circuits function.

Table 3. Test Circuit Characteristics and Results

\begin{tabular}{|c||c|c|c|}
\hline $\begin{array}{c}\text { RSFQ } \\
\text { Gate }\end{array}$ & $\begin{array}{c}\text { No. biases } \\
\text { to bus }\end{array}$ & $\begin{array}{c}\text { No. of } \\
\text { Junctions }\end{array}$ & Critical Margin \\
\hline \hline Splitter & 4 & 13 & $\pm 34 \%$ I $_{\text {bus }}$ \\
\hline AND & 8 & 26 & $\pm 12 \%$ I $_{\text {bus }}$ \\
\hline OR & 6 & 24 & $\pm 17 \% \mathrm{I}_{\text {in }}$ B \\
\hline XOR & 6 & 23 & $+9(-37 \%) \mathrm{I}_{\text {out }}$ \\
\hline half & 18 & 45 & 0 \\
\hline full & 61 & 107 & 0 \\
\hline
\end{tabular}

The circuits typically could be operated at the same bias conditions after thermal cycling, but occasionally the bias conditions changed due to trapped flux in the circuit. Sometimes a read SQUID would be in the opposite flux state or its threshold would be reduced for the same bias conditions, indicating the presence of trapped flux. Trapped flux either reduced margins or made the circuit nonfunctional.

\section{CONCLUSION}

Correct operation of the primary RSFQ logic circuits has been experimentally demonstrated at low speeds. The experimental test circuits have fair margins as compared to simulations, considering the use of bus biasing and fabrication uniformity. The impact of trapped flux on the circuits is clearly detrimental and requires further investigation. The more complex RSFQ functions on the chip, the half adder and full adder, were not successfully operated, even though the input and output circuits functioned properly. It is not clear whether this is a direct result of increased circuit complexity, decreased margins due to the single bus line, or some other difficulty.

\section{ACKNOWLEDGMENTS}

This research was supported in part by DARPA order No. 6882. K.C. Gilbert's support with the computer simulations was greatly appreciated.

\section{REFERENCES}

[1] K.K. Likharev, O.A. Mukhanov, and V. K. Semenov, "Resistive single flux quantum logic for Josephsonjunction digital technology," in $S Q U I D^{\prime} 85$ Superconducting Quantum Interference Devices and their Applications, eds., H.D. Hahlbohm and H. Luebbig, Walter de Gruyter \& Co., Berlin, 1985.

[2] K.K. Likharev, O.A. Mukhanov, and V. K. Semenov, "Ultimate performance of RSFQ logic circuits," IEEE Trans. Magnetics, vol. 23 pp. 759-762, Mar. 1987.

[3] K. K. Likharev and V. K. Semenov, "RSFQ logic/memory family: a new Josephson-junction technology for sub-terahertz-clock-frequency digital systems," IEEE Trans. Appl. Supercon., vol. 1, pp. 328, March 1991.
[4] C. A. Hamilton and K. C. Gilbert, "Margins and Yield in Single Flux Quantum Logic," IEEE Trans. Appl. Supercon., vol. 1, pp. 157-163, December 1991.

[5] V.K. Kaplunenko, M.I. Khamipov, V.P. Koshelets, K.K. Likharev, O.A. Mukhanov, V.K. Semenov, I.L. Serpuchenko, A.N. Vystavkin, "Experimental Study of the RSFQ Logic Elements," IEEE Trans. Magnetics, vol. 25 pp. 861-864, Mar. 1989.

[6] C. J. Burroughs and C. A. Hamilton, "Automated Josephson Integrated Circuit Test System," paper EOB10 , submitted to this conference (ASC 92). 